\title{
A Small-Area Scintillation Hodoscope Used for Components Test of MPD Detector in NICA Experiment
}

\author{
Madalina Cruceru, $^{1, *}$, Ilie Cruceru ${ }^{1}$, Anatoly Litvinenko ${ }^{2}$, Liviu Ciolacu ${ }^{1}$ \\ ${ }^{1}$ Department of Applied Nuclear Physics, HoriaHulubei National Institute for R\&D in Physics and Nuclear Engineering, \\ Magurele, RO-077125, Romania \\ ${ }^{2}$ Joint Institute Nuclear Research, Dubna 141980, Moscow Region, Russia \\ *Corresponding Author:madalina.cruceru@nipne.ro
}

Copyright (C) 2013 Horizon Research Publishing All rights reserved.

\begin{abstract}
This hodoscope is a position sensitive detector used in physics experiments at accelerators, for determination of position resolution of particles generated in nuclear interactions at high energy $\left(\sqrt{\mathrm{S}_{\mathrm{NN}}}=3-11 \mathrm{GeV}\right)$. This detector is a new type of device which uses plastic scintillators strips coupled to Hamamatsu PIN photodiodes. Usually in this kind of experiments are used position-sensitive devices on base of silicon strips and/or plastic scintillators coupled to photomultipliers(PMT). The first is very good but raises problems in construction, and the second operates at high voltage, resolution is high and its characteristics are affected by magnetic fields. The biggest advantage of the proposed hodoscope consists in easy construction, his characteristics is not influenced by high magnetic fields and has good performance in position resolution $(<5 \mathrm{~mm})$. This performance is obtained using an associated electronics which assures a multiparametric determination. The detector structure is made from two perpendicular planes of plastic scintillator strips, each having eight elements. Every strip is coupled to very small charge sensitive preamplifiers.
\end{abstract}

Keywords Plastic Scintillator, Silicon Strip, Response, Efficiency, Position Resolution

\section{Introduction}

The hodoscope with plastic scintillators and PIN photodiodes is a position sensitive detector used in experiments of nuclear physics at high energy (in relativistic nuclei collisions and heavy ions collisions). This hodoscope is a small-area detector which determinates the position resolution of the particles which appear in nuclear interactions at NICA(Nuclotron-based Ion Collider fAcility) for MPD(Multi Purpose Detector) proposed for the implementation at JINR (Dubna).

At NICA energies $\sqrt{S_{N N}}=3-11 \mathrm{GeV}$, this system is used to study the properties of excited hadronic matter produced from nuclear interactions. The experiments which will be performed with this type of hodoscope are experiments with fixed target at Nuclotron.

Usually, in this kind of experiments are used position-sensitive devices with plastic scintillators and photomultipliers tubes $[1,2,3]$ with lightguides. The position resolution is high and the hodoscope operates at high voltage because of the photomultipliers tubes. PIN photodiodes operates at low voltage.

Recently, have been used avalanche photodiodes [4] which take the scintillation light from the plastic scintillation pads.

Similar hodoscopes(with fiber optic light collection) but with large area can be used for cosmic ray muons detection [5]. They provide detection of cosmic ray muons in real-time.

The hodoscope used in NICA Experiment will test MPD Detector, detecting all types of particles generated at high energy.

A position sensitive detector, hodoscope can determine the xy coordinate of the detected particle. Like with the cylindrically detector [6] this detector type hodoscope uses the PIN photodiode toconvert the light generated in scintillation material in electric charges.

In this paper is presented only the hodoscope structure, not the associated electronics with which our hodoscope operates.

Also, in this paper we give only the preliminary results obtained in laboratory.

\section{Hodoscop Position in MPD}

Our hodoscope will be one of the MPD subsystems. It will be placed in front of the ZDC(Zero Degree Calorimeter) and will determinate the position resolution of the particles generated in nuclear interactions between beam and fixed target which interacts with ZDC. Particularly, collisions of heavy nuclei have a complicated space-time structure. To study this we need a universal detector which takes into 
account the impact parameter. Our hodoscope will do this.

\section{Hodoscope Description}

The hodoscope performed in our laboratoryis a small device which has 16plastic scintillator strips, 8 in the horizontal plane and 8 in the vertical plane, each strip having dimensions of $300 \times 10 \times 5 \mathrm{~mm}^{3}$.Every scintillator strip made from plastic scintillator material type BC 430 (Bicron Corporation), is coupled to a PIN Hamamatsu photodiode (Figure 1.).

The hodoscope is mounted on a dedicated support (Figure 2.) which can have the possibility to modify the position on vertically.

The strips are fixed on a metallic frame. Every strip is wrapped in Tyvek paper and black masking paper.The light readout is done by PIN photodiodes Hamamatsu with $5 \times 5$ $\mathrm{mm}^{2}$ active area. Every photodiode is coupled to a charge sensitive preamplifier. The main advantages of the photodiodes are: very compact size, low bias voltage, relative low price, gain comparable to PMT, insensitive to magnetic field. The preamplifier is very small.Our hodoscope is fixed on a wooden support which will make it to be easy to put in the experiment in front of the ZDC. As we know the hodoscope will determinates the position coordinates of the spectators which interact withZDC. The spectators are protons, neutrons or nuclei fragments with different ratio of $\mathrm{Z} / \mathrm{A}$. In this way when the magnetic field is constant (about $0.5-0.7 \mathrm{~T}$ ) and directed along beam, the spectator trajectory is a spiral.

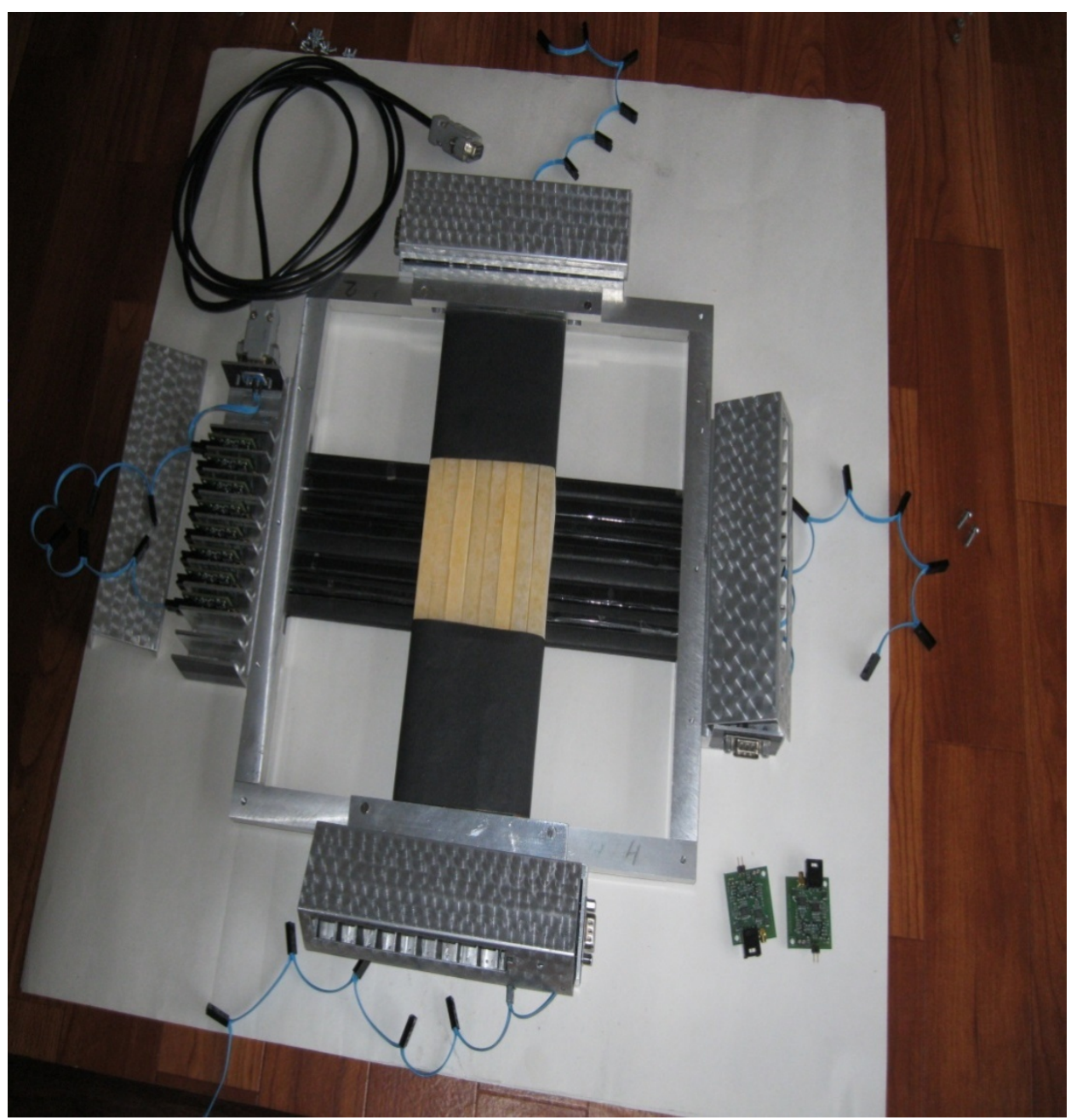

Figure 1. Hodoscope ensemble view 


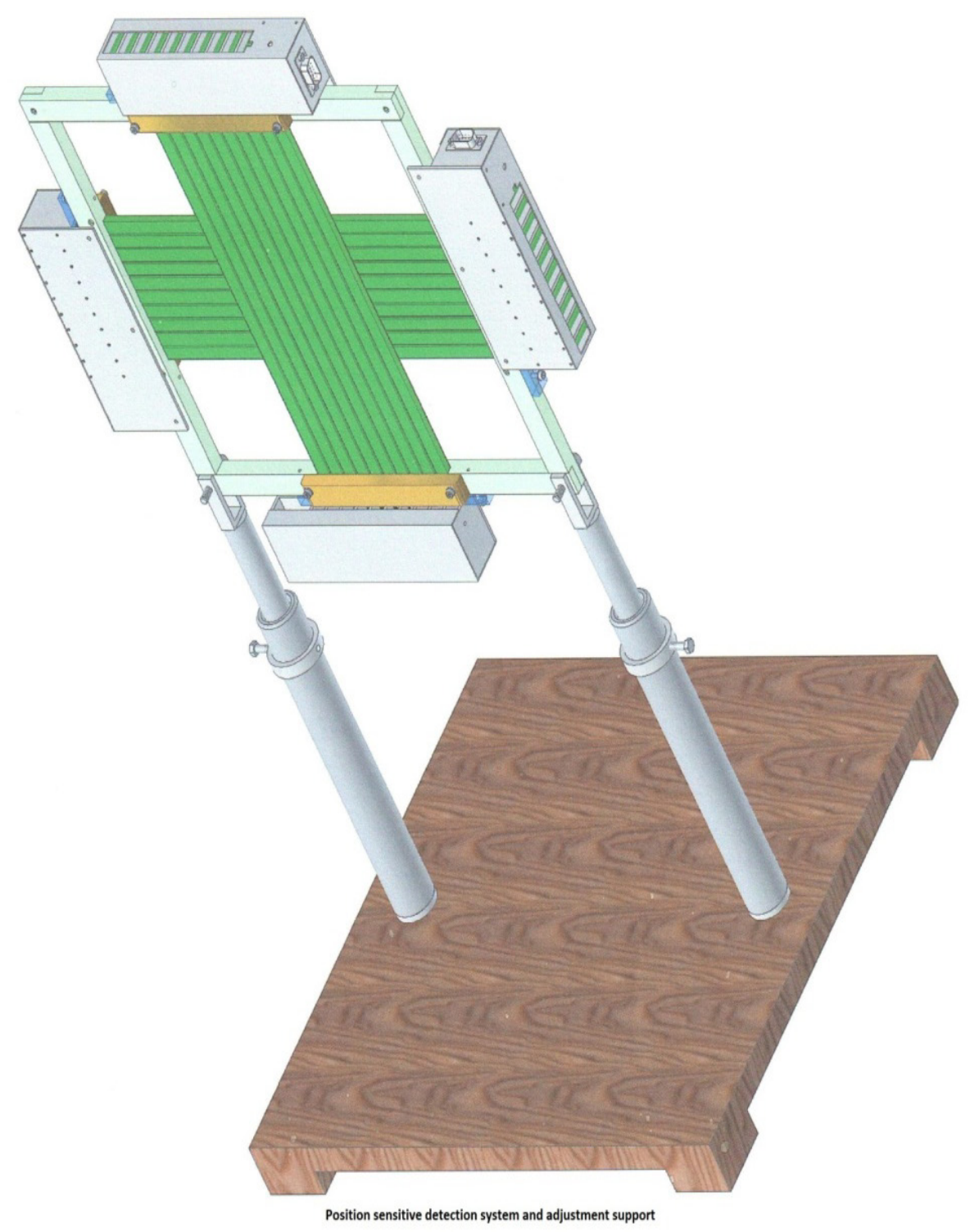

Figure 2. Hodoscope with adjustment support

\section{Experimental Results}

We tested this hodoscope with two radioactive sources: Co-60 and (Sr-Y)90. We did measurements for all hodoscope surfaces. All preamplifiers give a response to radiation. The information obtained by the left side preamplifiers is similar to the right side preamplifiers responses.

The information about response is registered with some counters after the signals were passing in leading edge discriminator (LED).

The curve of response of each detector element is presented in Figure 2:

In the Figure 3, we observe a variation of response in length of the strip. This is due to light attenuation and reflection along the strip. In the Figure 3.,L is the detector length and $\mathrm{N}$ is the total number of counts per seconds.

If we don't work in MIP's regime, the hodoscope efficiency will be $60 \%$. For one strip we obtain this variation of efficiency with distance source-PIN photodiode(Figure 3.):

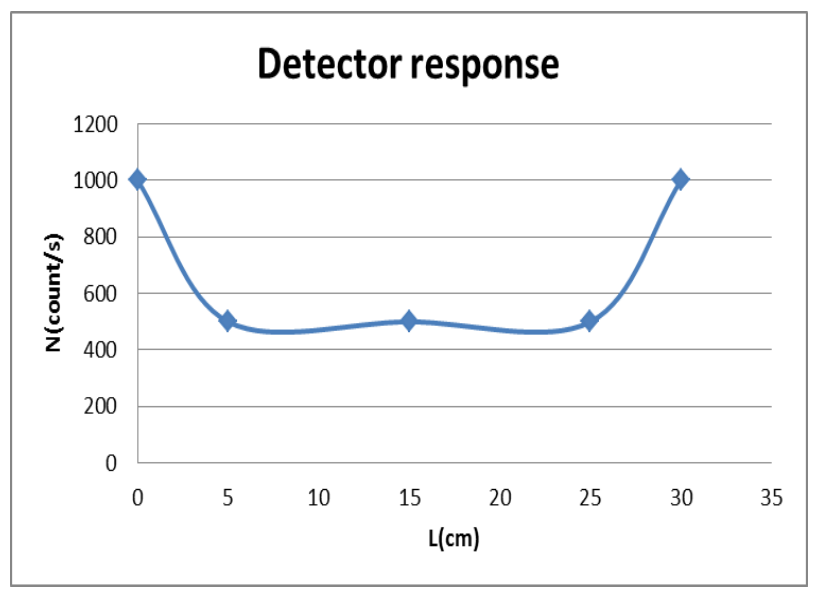

Figure 3. Response curve for hodoscope with radioactive source of $(\mathrm{Sr}-\mathrm{Y}) 90$ 


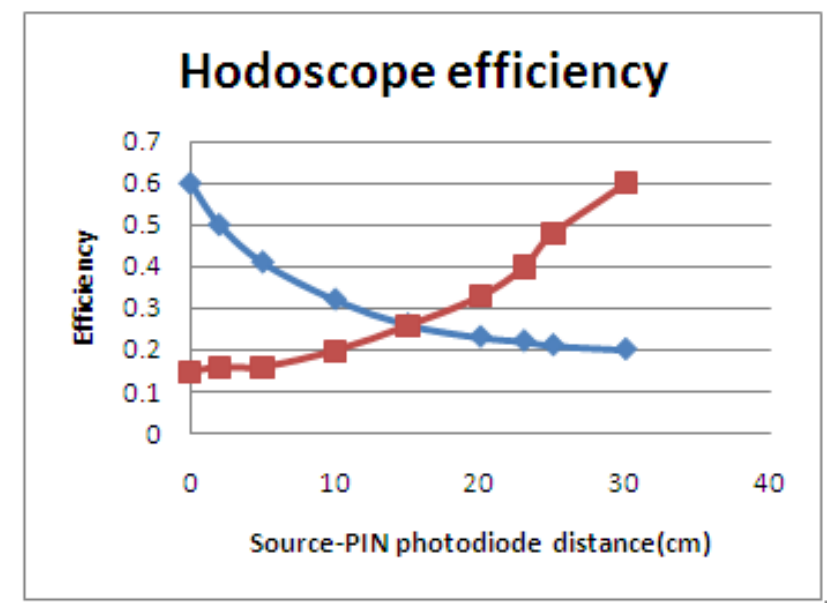

Figure 4. Hodoscope efficiency for one strip

In the Figure 4, with blue curve is represented the efficiency when the radioactive source is moving from the left end of the strip to the right end of the strip. Red curve represents the efficiency when the radioactive source is moving from the right end of the strip to the left end of the strip.

The performance of the hodoscope for many types of particles used for tests (electrons, gamma, deuterons and muons) is presented in Figure 5. In this figure we have the energy loss distribution vs. number of particles on $\mathrm{x}$ detector axis. Because the plane $\mathrm{x}$ of the hodoscope is similar with the $y$ plane of it, we present only this distribution in plane $x$.

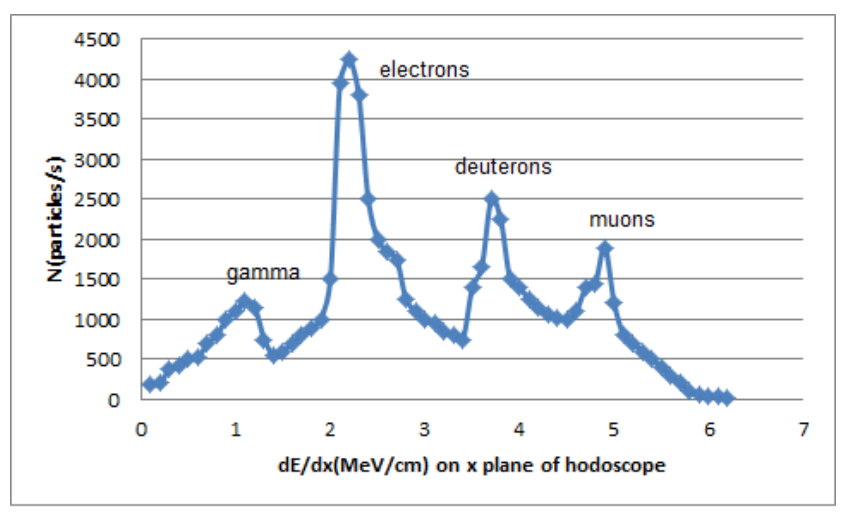

Figure 5. Energy loss distribution vs. number of particles for plane $\mathrm{x}$ of hodoscope

\section{Conclusions}

This type of detector is used in NICA-MPD physics program for searching the dense and hot baryonic matter. The efficiency of the hodoscope can touch value of $90 \%$ in MIP's (minim ionization particles)regime. The position resolution is smaller than $5 \mathrm{~mm}$. The hodoscope response is almost uniformly on all surfaces and has maximum values on the edges.

The hodoscope efficiency is not the same for all length of the strip because of the irregular structure of the scintillator strips.

The performance of this detector is very good for electrons, protons, ions and heavy ions.

\section{Acknowledgements}

The work is done in the framework of the research contract no.08626319/1220557-74 with JINR Dubna, Russia. This hodoscope was required for NICA-MPD Experiment.

\section{REFERENCES}

[1] B.Chiladze,S.Dymov, R.Esser, M.Hartmann, R.Koch, V.Komarov, A.Kulikov, G.Macharashvili, S.Merzlyakov, M.Nioradze, A.Petrus, B.Rimarzig, R.Schleichert, H.Seyfarth, A.Volkov, B.Zalikhanov, N.Zhuravlev, The forward detector of the ANKE spectrometer. Scintillation and Cherenkov hodoscopes, Pleiades Publishing Ltd., Particles and Nuclei, Letters. Vol.4, 95-100, 2002.

[2] M.W.Collins, A.E.Groome, Performance of a small, fast, high gain photomultiplier tube for use with scintillation counter hodoscope array in high energy physics experiments, North-Holland Publishing CO., Nuclear Instruments and Methods Vol.79, 283-285, 1970.

[3] M.W. Collins, A.E.Groome, P.R.Norton, Scintillation counter hodoscope array for accurate location of particle trajectories in magnetic spectrometers, North-Holland Publishing CO., Nuclear Instruments and Methods 117(1974) 339-344.

[4] E.N.Gushkin,V.F.Kurshetsov, V.A.Lebedev, V.I.Romanovsk iy, V.K.Semenov, S.N.Filippov, A.A.Khudyakov, Matrix hodoscopes for the Oka(protvino) and NA62(SPS, CERN) Experimental Setups, Pleiades Publishing, Ltd., Physics of Atomic Nuclei, Vol.74, 783-787, 2011.

[5] N.V.Ampilogov,I.I.Astapov, N.S.Barbashina, V.V.Borog, D.V.Chernov, A.N.Dmitrieva, K.G.Kompaniets, A.A.Petrukhin, V.V.Shutenko, A.I.Teregulov, I.I.Yashin, Large area scintillation muonhodoscope for monitoring of atmospheric and heliospheric processes, Copernicus Publications on behalf of the ArbeitsgemeinschaftExtraterres trischeForschunge.V., Astrophysics and Space Sciences Transaction Vol.7, 435-438, 2011.

[6] MadalinaCruceru, D.Bartos, I.Cruceru, G.Caragheorghiopol, O.G.Duliu, A.Barborica, P.Cristea, C.M.Niculae, R.C.Bobulescu, A coordinate sensitive detector for particles generated in high energy reactions, Publishing House of the Romanian Academy, Romanian Reports in Physics, Vol.61, 513-521, 2009. 